\title{
CALCULATION OF PROVISIONS USING CREDIBILITY THEORY
}

M. A. Pereira Cabral

AND

J. M. Afonso Garcia

Lisbon

I

\section{PROVISIONS}

\section{INTRODUCTION}

The increasing difficulties facing the insurer when he measures certain risks and fundamentaly when that measurement has to be carried out "a priori" are not easily overcome. Thus, the greater part of the insurance companies still use in numerous branches subjective methods of assessing a risk, which unfortunately do not lead to the best results. Especially in certain risks with undefined characteristics, the problem becomes more accentuated to the point of being practically impossible to class risks whose behaviour would be homogeneous.

For example we can analyse the numerous problems of this nature arising in the motor insurance in all countries in assessing the combined risk motor-car and driver.

Numerous solutions have been proposed and amongst them a system of premiums adapted to the actual risk and very especially the credibility premiums represent a fundamental step in the evaluation of these types of risks, for they have an advantage of at least as experience increases assuming an authentic measurement "a posteriori" of the true degree of risk which they cover.

In all, once more we can look at the motor insurance as an example, the serious problem of drawing up premiums in the first years of duration of the contracts, continues, despite the fact that some precautionary measures have been taken into account, without on the whole being effective, in the larger number of cases. We believe that in certain cases, one mode of action remains before the 
insurer to overcome these difficulties, the charging of provisional premiums which we will refer to "provisions", which as time elapses would be adjusted to the real value of the risk and the setting up of a system of "surplus distribution" which would permit the excess premium to fall back upon the policy-holder.

The establishing of provisions is common practice amongst insurers, although it is due not to the lack of knowledge of the nature of the risk but to its dimention.

As we have already mentioned it seems possible to generalize the application of provisions to all risks whose claim frequency in a short interval of time will indicate its true risk grade.

The method which we will elaborate consists essentially of an introduction of the credibility theory into the calculation of provisions, closely following the development of that theory by prof. Bühlmann with adaptations thought necessary.

\section{Credibility Provisions}

2.I. If we consider a provision to act as a "buffer" to the eventual bad claim, it seems that we should base its determination upon the deviation, claims have from the mean value that normally serve as the base to the calculation of premiums.

We can not proceed with the development of the proposed method without defining more precisely the basic characteristics which a provision should have.

Thus a provision:

I) should not in any circumstance be lower than the average premium of the risk class to which the risk belongs.

2) should contain security margin based on the positive deviation to the mean value.

3) should take into account the bad claims of the risk class and the bad claims observed in that risk in the past.

Further on we will analyse these characteristics in greater detail which although they can be slightly subjective, have the advantage of respecting the base concept of "mean positive deviation".

2.2. Following prof. Bühlmann, we consider a collective $\Theta$ characterised as a set of risks with paremeter $\theta$ and by the distribu- 
tion function $U(\theta)$-structure function. Let $\left\{S_{1}, S_{2}, \ldots, S_{n}\right\}$ be a set of independent random variables with identical D.F. defined in each year as the total claims of a certain risk $\theta$ and $G^{\theta}(x)$ its respective $D . F$.

We also consider the distribution function $G(x)$ for the collective $\Theta$ defined by the equation

$$
G(x)=\int_{\Theta} G^{\theta}(x) d U(\theta)
$$

If we call $\mu(\theta)$ and $\mu$ the respective mean values we will obtain

$$
\mu(\theta)=\int_{0}^{+\infty} x d G^{\theta}(x) \quad \mu=\int_{0}^{+\infty} x d G(x)
$$

The relation $\mu=E[\mu(\theta)]$ being valid.

Consider a conditional random variable $Y / S \geqslant \mu$ representing the positive deviation in relation to $\mu$, or $Y=S-\mu$ if $S \geqslant \mu$.

Its D.F. will be for a certain risk $\theta$

$$
F^{\theta}(y)=\frac{\int_{\mu}^{y+\mu} d G^{\theta}(x)}{\int_{\mu}^{+\infty} d G^{\theta}(x)}=\frac{G^{\theta}(y+\mu)-G^{\theta}(\mu)}{I-G^{\theta}(\mu)}
$$

Being $\delta(\theta)$ the respective mean value, we have:

$$
\delta(\theta)=\int_{0}^{+\infty} y d F^{\theta}(y)
$$

For a risk taken at random from the collective we obtain the same equation

$$
\begin{gathered}
F(y)=\frac{G(y+\mu)-G(\mu)}{\mathrm{I}-G(\mu)} \\
\text { If } \left.\left.\delta=\int_{0}^{+\infty} y d F(y) \text { we can obtain } \quad \delta \neq E[\delta] \theta\right)\right]
\end{gathered}
$$

In effect,

$$
\delta=\int_{0}^{+\infty} \frac{y}{\mathrm{I}-G(\mu)} d G(y+\mu)=\frac{\mathrm{I}}{\mathrm{I}-G(\mu)} \int_{0}^{+\infty} y d \int_{\theta} G^{\theta}(y+\mu) d U(\theta)
$$




$$
\begin{gathered}
=\frac{\mathrm{I}}{\mathrm{I}-G(\mu)} \int_{\Theta} \int_{0}^{+\infty} y d G^{\theta}(y+\mu) d U(\theta) \\
=\frac{\int_{\Theta} \delta(\theta)\left[\mathrm{I}-G^{\theta}(\mu)\right] d U(\theta)}{\int_{\Theta}\left[\mathrm{I}-G^{\theta}(\mu)\right] d U(\theta)} \quad(\mathrm{A})
\end{gathered}
$$

As $\int_{\Theta} \delta(\theta)\left[\mathrm{I}-G^{\theta}(\mu)\right] d U(\theta) \neq \int_{\Theta} \delta(\theta) d U(\theta) \cdot \int_{\Theta}\left[\mathrm{I}-G^{\theta}(\mu)\right] d U(\theta)$ the expression (A) is different from $E[\delta(\theta)]=\int_{\Theta} \delta(\theta) d U(\theta)$

If we have defined $F(y)$ as the weight distribution of $F^{\theta}(y)$ as we defined $G(x)$, that is if $F(y)=\int_{\Theta} F^{\theta \theta}(y) d U(\theta)$, we would have obtained the equality $\delta=E[\delta(\theta)]$. We did not use this definition in order to continue using the following expression

$$
F(y)=\frac{G(y+\mu)-G(\mu)}{\mathbf{I}-G(\mu)}
$$

Let us consider the function $\pi(\theta)=\delta(\theta) \cdot P\left(x^{\theta} \geqslant \mu\right)$ and for $\pi$ the product $\delta . P(X \geqslant \mu)$. We can verify the relation $\pi=E[\pi(\theta)]$.

$$
\text { In effect, } \begin{aligned}
\pi & =\int_{0}^{+\infty} y d F(y) \cdot P(X \geqslant \mu)=\int_{0}^{+\infty} y d G(y+\mu) \\
& =\int_{\Theta} \int_{0}^{+\infty} y d G^{\theta}(y+\mu) d U(\theta)=\int_{\Theta} \delta(\theta) \cdot P\left(X^{\theta} \geqslant \mu\right) d U(\theta) \\
& =E[\pi(\theta)]
\end{aligned}
$$

this equality is fundamental in the theory which we shall develop.

2.3. We can now go on with the analytical expression of the provision based on the characteristics defined in 2.I.

Consider a random risk of the collective. Two complementary situations can occur, in reference to the total $x$, during the period for which we want to determine the provision.

$$
\begin{aligned}
& \text { I } x<\mu \\
& 2 x \geqslant \mu
\end{aligned}
$$

If we admit that in the first situation $\mu$ is a sufficient provision and that in the second situation we have to fall back on the value $\mu+\delta$, we can obtain a value for the provision by weighting these 
values by their respective probabalities of occurence. Thus, we have:

$$
\begin{aligned}
P R & =\mu \cdot P(x<\mu)+(\mu+\delta) \cdot P(x \geqslant \mu) \\
& =\mu+\delta \cdot P(x \geqslant \mu)=\mu+\pi .
\end{aligned}
$$

It is possible to see that a so determined provision respects the basic principles $I$ and 2 defined in $2 . I$.

In the same manner for a given risk $\theta$ we have

$$
P R(\theta)=\mu+\delta(\theta) \cdot P\left(x^{\theta} \geqslant \mu\right)=\mu+\pi(\theta) .
$$

It would be interesting to verify the relation

$$
P R=E[P R(\theta)]
$$

which follows from the equality $\pi=E[\pi(\theta)]$ already proven.

2.4. As the parameter $\theta$ associated with each risk is unknown it is not possible to estimate $\pi(\theta)$ directly, thus we have to obtain the value of this function by an approximation using credibility theory.

Consider the principle $\mathfrak{F}$ or in other words the method of calculation of $\pi$ which would permit the insurer to obtain a compensation for eventual bad claims of any risk. We thus have $\pi=\mathfrak{h}[F(x)]$

In the same manner for a given risk $\theta$ we have $\pi(\theta)=\mathfrak{h}\left[F^{\theta}(x)\right]$

Let $\left\{D_{1}, D_{2}, \ldots, D_{n}\right\}$ be a set of random variables corresponding to the "positive deviations" of the years $I, 2, \ldots \ldots, n$ and $D_{n+1}$ corresponding to the deviation of the following year.

If $S$ be the sum of claims, the variables $D$ will have the following form

$$
\begin{array}{ll}
D=(S-\mu) \cdot P(X \geqslant \mu) & \text { if } S \geqslant \mu \\
D=0 & \text { if } S<\mu
\end{array}
$$

We note that $\pi=\delta . P(X \geqslant \mu)=E(D)$ and that the principle $\mathfrak{H}$ associates the random variable $D$ to its respective mean value.

Thus the application of $\mathfrak{H}$ to the variable $\pi(\theta)$ will give

$$
\pi^{*}=\mathfrak{H}[\pi(\theta)]=\int_{\theta} \pi(\theta) d U(\theta)=E[\pi(\theta)]=\pi
$$

We thus can see that the principle $\mathfrak{H}$ is iterative, thus the application of the base theorem of credibility theory will allow us to establish the following relationship:

$$
D_{n+\mathbf{1}}\left(D_{1}, D_{2}, \ldots, D_{n}\right)=\mathfrak{S}\left[D / D_{1}, D_{2}, \ldots, D_{n}\right]
$$


instead of the equation

$$
D_{n+1}\left(D_{1}, D_{2}, \ldots, D_{n}\right)=\mathfrak{h}\left[\pi(\theta) / D_{1}, D_{2}, \ldots, D_{n}\right]
$$

which alone would not give the solution to the problem as $\theta$ continues to be an unknown quantity.

2.5. In accordance with the principle $\mathfrak{H}$, we want to estimate the value of

Let

$$
E\left[\pi(\theta) / D_{1}, D_{2}, \ldots, D_{n}\right]
$$

$$
\bar{D}=\frac{D_{1}+D_{2}+\ldots+D_{r}}{r} \cdot \frac{r}{n} \quad r \leqslant n
$$

where $r$ is the number of observed "positive deviations".

In accordance with the definition of $D$ we can also write the equation

$$
\bar{D}=\frac{D_{1}+D_{2}+\ldots+D_{n}}{n}
$$

Following prof. Bühlmann we shall linearize the expected value above indicated by the relation:

$$
E\left[\pi(\theta) / D_{1}, D_{2}, \ldots, D_{n}\right]==a+b \bar{D}
$$

The constants $a$ and $b$ will be determined by the minimization of the value of

$$
\begin{gathered}
E\left[\left\{E\left[\pi(\theta) / D_{1}, D_{2}, \ldots, D_{n}\right]-(a+b \bar{D})\right\}^{2}\right] \\
=\int\left\{E\left[\pi(\theta) / D_{1}, D_{2}, \ldots, D_{n}\right]-(a+b \bar{D})\right\}^{2} d W\left(D_{1}, D_{2}, \ldots, D_{n}\right)
\end{gathered}
$$

As we can see the constants $a$ and $b$ thus determined also minimize the value

$$
\begin{aligned}
& E\left[\{\pi(\theta)-(a+b \bar{D})\}^{2}\right] \\
& \text { In effect } E\left[\{\pi(\theta)-(a+b \bar{D})\}^{2}\right]= \\
&=E\left\lceil\left\{\pi(\theta)-E\left[\pi(\theta) / D_{1}, \ldots, D_{n}\right]+E\left[\pi(\theta) / D_{1}, \ldots, D_{n}\right]-(a+b \bar{D})\right\}^{2}\right] \\
&=E\left[\left\{\pi(\theta)-E\left[\pi(\theta) / D_{1}, \ldots, D_{n}\right]\right\}^{2}\right]+E\left[\left\{E\left[\pi(\theta) / D_{1}, \ldots, D_{n}\right]-\right.\right. \\
& \quad 2 E\left[\pi(\theta) \cdot E\left[\pi(\theta) / D_{1}, \ldots, D_{n}\right]-\pi(\theta) \cdot(a+b \bar{D})-\right. \\
& \quad-\left\{E\left[\pi(\theta) / D_{1}, \ldots, D_{n}\right]\right\}^{2}+ \\
&\left.+E\left[\pi(\theta) / D_{1}, \ldots, D_{n}\right] \cdot(a+b \bar{D})\right]=(A)+(B)
\end{aligned}
$$


Here we have written for short:

$$
\begin{aligned}
& (A)=E\left[\left\{\pi(\theta)-E\left[\pi(\theta) / D_{1}, \ldots, D_{n}\right]\right\}^{2}\right] \\
& (B)=E\left[\left\{E\left[\pi(\theta) / D_{1}, \ldots, D_{n}\right]-(a+b \bar{D})\right\}^{2}\right]
\end{aligned}
$$

once that by hypothesis $E\left[\pi(\theta) / D_{1}, \ldots, D_{n}\right]=a+b \bar{D}$.

But as $(A)$ is fixed, minimizing $(B)$ is equal to minimizing $(A)+$ $(B)$.

It also happens that the value $a+b \bar{D}$ thus obtained also minimizes

$$
E\left[\{b[\pi(\theta)-\bar{D}]\}^{2}\right]+E\left[\{(\mathrm{I}-b) \pi(\theta)-a\}^{2}\right]
$$

once that

$E\left[\{\pi(\theta)-(a+b \bar{D})\}^{2}\right]=E\left[\left\{b[(\pi(\theta)-\bar{D}]+[(\mathrm{I}-b) \pi(\theta)-a]\}^{2}\right]\right.$.

We then conclude that $a=(\mathrm{I}-b) . E[\pi(\theta)]$, minimizes the previous expressions.

Substituting $a$ by its value we obtain

$$
b=\frac{\operatorname{Var}[\pi(\theta)]}{\operatorname{Var}[\bar{D}]}
$$

As it is not possible to estimate $\operatorname{Var}[\pi(\theta)]$ directly by statistics over the collective, we will obtain an expression for $b$ whose quantities will be easily estimated, assuming that we have a set on annual statistics.

As the independence of the variables $S_{1}, S_{2}, \ldots, S_{n}$ implies the independence of $D_{1}, D_{2}, \ldots, D_{n}$ we can write

$$
\begin{aligned}
& \operatorname{Var}(\bar{D})=(\mathrm{I} / n) E[\operatorname{Var}(D / \theta)]+\operatorname{Var}[E(D / \theta)] \\
& \operatorname{Var}(D)=E[\operatorname{Var}(D / \theta)]+\operatorname{Var}[E(\mathrm{D} / \theta)]
\end{aligned}
$$

From these expressions we can obtain the equation

$$
\operatorname{Var}[\pi(\theta)]=\frac{n \cdot \operatorname{Var}(\bar{D})-\operatorname{Var}(D)}{n-\mathrm{I}}
$$

in which only figure quantities estimable from the collective, thus

$$
b=\frac{n \cdot \operatorname{Var}(\bar{D})-\operatorname{Var}(D)}{(n-\mathrm{I}) \cdot \operatorname{Var}(\bar{D})}
$$


We can then obtain

$$
E\left[\pi(\theta) / D_{1}, D_{2}, \ldots, D_{n}\right]=b \bar{D}+(\mathrm{I}-b) \pi
$$

and the provision for the year $(n+\mathrm{I})$ will take on the following form:

$$
P R_{n+1}=\mu+b \bar{D}+(\mathrm{I}-b) \pi
$$

As we can see this expression obeys the base principle 3 referred in 2.I.

\section{Surplus Distribution}

The levy of provisions or premiums with excess margins of security can not be admitted on equitable principles without being associated with the distribution of surplus to the policy-holders.

This distribution should especially take into account the individual results of each contract and also the global results of the portfolio to which each of the contracts belongs.

On the other hand, the accumulation of reserves obliges the insurer to consider a financial compensation as a just payment for the values the insurer "deposited" in excess with the insurance company.

There are numerous schemes already in existence that distribute surpluses, in general any scheme that is applicable to life assurance is also applicable with slight modifications to any other contingencies.

In this system we are about to describe and which will be the practical example of this paper, we opted for a fixed triannual period in which bonuses are distributed amongst policy-holders independent of the initial age of their respective policies but the initial date of the policy was taken into account in the scheme.

3.I. Consider a fund $F$ updated annually by the positive or negative balance of the following account.

\section{Credit}

- Previous years balance. (If positive)

- $\Sigma(P R-\mu)$, the excess levied during the year in all the collective 
- K.L/I0o, $K$ being a determined percentage and $L$ the profit generated by the portfolio. (If positive)

- Interest on the above items calculated for the end of the year with a rate equal to the net investment rate of the respective branch.

\section{Debit}

- Previous years balance (If negative)

- (- $L$ ) (If $L$ negative)

- Surplus eventually distributed during the year

- Interest on the above mentioned items.

3.2. Triannually the sum $M$ is distributed amongst the policyholders. That sum represents a percentage of $F$ not smaller than a minimum value previously established. The remainder will be left in the fund as a stabilizing reserve.

In the practical exemple we used $70 \%$ as the pre-established percentage $(M=0.7 . F)$.

The distribution of $M$ for contracts with different initial dates is directly proportional to their respective provisions, that is representing the total of the levied provisions, and the sums to be distributed amongst the insured with policies belonging to generation $I$, by $P R(I)$ and $F(I)$ we then have

$$
F(I)=M \frac{P R(I)}{\Sigma} \frac{P R(I)}{P R}
$$

The distribution of $F(I)$ amongst the policies may, and should be directly proportional to the theoretical balance of the policy.

For such procedure it is necessary to grade the various contracts of each generation, by their theoretical balances.

Consider a certain risk $\theta$ aged $n$ years whose theoretical balance we want to determine during the last $k$ years $(k \leqslant n)$.

If we consider that in each year $P R(\theta)-P_{n+1}(\theta)$ where $P R(\theta)$ is the levied provision, and $P_{n+1}(\theta)$ the credibility premium for the $(n+\mathrm{I})$ th year, based on all the past experience of that risk, we obtain a "more probable" value for the annual theoretical result which we want to determine.

Thus

$$
\sum_{i=1}^{k}\left[P R_{i}(\theta)-P_{n+1}(\theta)\right] r^{k-i}
$$


$r$ being the financial actualisation factor, which will give us a good idea of the theoretical result of that risk during the $k$ years. $\theta$ is an unknown, but we know $P R_{i}$ and we can find $P_{n+1}$.

As we mentioned above each policy will be registered in its respective grade according to its theoretical result, and thus we can easily distribute the total amount $F(I)$ amongst the different policies.

Thus, if $N(I, K)$ and $V(I, K)$ represent the mentioned values, and if $X(I, K)$ represent the sum to be distributed for each risk of generation $I$ belonging to grade $K$, we have

$$
X(I, K)=\frac{F(I) \cdot V(I, K)}{\sum_{K} N(I, K) \cdot V(I, K)}
$$

4. We can not consider as final the defined scheme without some comments anticipating criticism to which a paper of this nature will be subjected, given the inexperience of the authors on one hand and the rather subjective nature of the used concepts, on the other.

4.I. The basic principles for calculation of provisions can be quite different from those used. From a system of unique provision to be levied on all policies, to a system where the scaling of provisions is based on the past experience of each risk, one can imagine a multitude of more or less practical, or satisfactory systems.

4.2. The principle $\mathfrak{H}$ we can use in calculating "positive deviations" can be different from the one used, as in the case of calculation of premiums.

4.3. The actual scheme of surplus distribution is still more debatable, but this paper has no other end than to show a possible way in which the surplus can be distributed, minimizing the administrative costs. A participation at the end of $k$ years of duration of each policy would be more logical but would undesirably stress branches already rather complex as regards administration.

4.4. Finally we do not want to leave out the difficulties and objections that would be raised in any country to a scheme of this nature, given its social and even legal implications. Also it would have the advantage of more equitable insurance contracts. On the 
other hand a system directly related with the actual risk would make the policy-holders more responsable for their claims, which already happens with credibility premiums.

\section{APILICATION TO 3rd. PARTY MOTOR INSURANCE}

I. We wish to calculate the provisions that would substitute the premiums in a portfolio of motor car insurance and using a simulation of that portfolio during 3 years, to determine the value of the surplus to be distributed to each policy-holder in order to compare the values paid in effect with the values that should have been paid under a system of credibility premiums.

Statistical data that we could obtain was not sufficient for this work as it was only related to annual observations.

Thus we were compelled to simulate our statistical data composed of 2500 fictitious policies "observed" during 4 years, in order to be able then to simulate the working of a provision scheme based on the exposed theory.

I. I. Statistical data obtained from a real portfolio.

a) Data obtained from a sample of 5000 policies.

\begin{tabular}{lccccc}
\hline $\begin{array}{l}\text { No. of } \\
\text { claims }\end{array}$ & o & I & 2 & 3 & 4 \\
\hline Frequency & 2913 & I532 & 38 I & I02 & 72 \\
\hline$E(K)=.5776$ & $\operatorname{Var}(K)=.6916$
\end{tabular}

b) Data obtained from a sample of rooo policies.

Total costs of claims are expressed in monetary units (ESCUDOS)

\begin{tabular}{|c|c|c|c|c|c|c|c|c|c|c|}
\hline $\begin{array}{l}\stackrel{+}{\Xi} \\
\underset{\Xi}{\Xi} \\
\underset{J}{\Xi}\end{array}$ & $\begin{array}{l}8 \\
\stackrel{8}{1} \\
\stackrel{H}{H}\end{array}$ & $\begin{array}{l}8: \\
8 \text { in } \\
\wedge V\end{array}$ & 욤 & 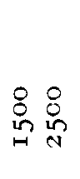 & $\begin{array}{l}0 \\
\stackrel{0}{0} \\
i \\
\end{array}$ & $\begin{array}{l}88 \\
8 \\
\text { in } \\
8\end{array}$ & $\begin{array}{ll}8 & 0 \\
8 & 8 \\
8 & 8 \\
10 & 10\end{array}$ & 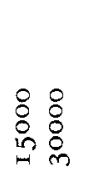 & \begin{tabular}{l}
8 \\
$\&$ \\
$\&$ \\
\hdashline
\end{tabular} & $\begin{array}{l}8 \\
8 \\
8 \\
1 \\
\wedge \\
-\end{array}$ \\
\hline Freq. & 2 & $3 \mathbf{I}$ & 262 & 203 & 289 & I 32 & 46 & I 7 & 8 & Io \\
\hline
\end{tabular}




\section{Claims Simulation}

2.I. We consider a set of 2500 policies and we generated for each of them, during 4 years, the number of accidents and their respective cost.

For simplicity we used the structure function

$$
U(\theta)= \begin{cases}0 & \text { for } \theta \leqslant .0776 \\ \theta-.0776 \text { for } .0776<\theta \leqslant 1.0776 & \text { for } \theta>1.0776\end{cases}
$$

whose mean value coincided with the mean value of our initial sample.

The random numbers were determined by the Lehmer's congruential method.

For each policy we randomly determined its $\theta$, which was maintained during the 4 years.

With the initial value $\theta$ we generated the number of claims that occurred during the first year in accordance with Poisson's D.F. with mean value $\theta .{ }^{1}$ )

The number of claims that occurred in the following years were determined by weighting the values generated by Poisson's law with the past experience of each policy by the following credibility expression.

$$
L=I[b \bar{K}+(\mathrm{I}-b) N(\theta)+.5]
$$

$\bar{K}$ being the mean value of the past experience and $N(\theta)$ the value genérated by Poisson's law.

The credibility coefficients that we used were based on initial data referred to in I.2 a) and they have the following values:

$$
.16, .28, .37 \text {. }
$$

Next in accordance with the distribution of the totals, $L$ values were generated and accumulated to give the annual total.

1) The generation of the number of claims by Poisson's law was carried out using the D. F. of the claim interoccurrence times wich are exponentially distributed with parameter $\theta$. 
2.2. Frequencies obtained after the generation program

\begin{tabular}{cccccc}
\hline $\begin{array}{r}\text { No. Claim } \\
\text { Year }\end{array}$ & o & I & 2 & 3 & 4 \\
\hline I & I 474 & 725 & 23 I & 63 & 7 \\
2 & 1485 & 732 & 218 & 59 & 6 \\
3 & I442 & 770 & 235 & 48 & 5 \\
4 & I 401 & 846 & 2 I0 & 37 & 6 \\
\hline
\end{tabular}

The calculated mean values were

\begin{tabular}{ccccc}
\hline Year & I & 2 & 3 & 4 \\
\hline MV & 2435 & 2259 & 2298 & 2287 \\
\hline
\end{tabular}

$M V=2320$

\section{Credibility Coefficients}

3.I. From the previous results we calculated with a different program the credibility weights for premiums and provisions using the following formulae

$$
\begin{aligned}
b_{P} & =\frac{\operatorname{Var}[\mu(\theta)]}{\operatorname{Var}[\bar{S}]} \\
b_{P R} & =\frac{\operatorname{Var}[\pi(\theta)]}{\operatorname{Var}[\bar{D}]}
\end{aligned}
$$

$\operatorname{Var}[\mu(\theta)]$ and $\operatorname{Var}[\pi(\theta)]$ were estimated by the expressions

$$
\begin{aligned}
& \operatorname{Var}[\mu(\theta)]=\frac{n \operatorname{Var}(\overline{S)}-\operatorname{Var}[S]}{n-\mathrm{I}} \\
& \operatorname{Var}[\pi(\theta)]=\frac{n \operatorname{Var}[\bar{D}]-\operatorname{Var}[D]}{n-\mathrm{I}}
\end{aligned}
$$

3.2. Given the slight variation in the variances we did not use directly the expression

$$
b=\frac{n \operatorname{Var}[\bar{X}]-\operatorname{Var}[X]}{(n-\mathrm{I}) \operatorname{Var}[\bar{X}]}
$$

We first estimated the variance values of $\mu(\theta)$ and $\pi(\theta)$ using the expressions defined in 3.I. 
3.3. Results

\begin{tabular}{rrrrr}
\hline $\mathrm{k}$ & $\mathrm{I}$ & 2 & 3 & \multicolumn{1}{c}{4} \\
\hline $\operatorname{var}(\bar{S})$ & $36.7 \mathrm{I}$ & 20.19 & 13.80 & I0.86 \\
$\operatorname{var}(\bar{D})$ & $3 \mathrm{I} .65$ & $\mathbf{1} 7.26$ & $\mathbf{1} \mathbf{I} .6 \mathrm{I}$ & 8.95 \\
\hline $\operatorname{Var}[\mu(\theta)]=2758$ & & & \\
$\operatorname{Var}[\pi(\theta)]=\mathrm{I} 480$ & & &
\end{tabular}

The credibility coefficients for the 2 nd. year were determined by polinomial extrapolation using the coefficients for the following years. It is interesting to note that they did not deviate significantly from the values

\begin{tabular}{ccccc} 
& $\frac{\operatorname{Var}[\mu(\theta)]}{\operatorname{Var}\left[S_{1}\right]}$ & \multicolumn{3}{|c|}{$\frac{\operatorname{Var}[\pi(\theta)]}{\operatorname{Var}\left[D_{1}\right]}$} \\
\hline $\mathrm{k}$ & 2 & 3 & 4 & 5 \\
\hline$b_{p}$ & .07 & .14 & .20 & .26 \\
$b_{p r}$ & .04 & .09 &. $\mathrm{I} 3$ &. $\mathrm{I} 7$ \\
\hline
\end{tabular}

4. Application of the credibility coefficients to a portfolio composed of 2500 policies with the following composition:

Generation I

Generation 2

Generation 3

Generation 4
I500 policies aged more than 3 years 200 policies aged 3 years 300 policies aged 2 years 500 policies aged I year

4.I. Results

All the following results were financially brought to the end of the 3 years period with the interest rate of $4.0 \%$

Claims/rooo

\begin{tabular}{|c|c|c|c|c|}
\hline $\begin{array}{l}\text { Year } \\
\text { Gen. }\end{array}$ & I & 2 & 3 & Total \\
\hline I & $36 \mathrm{I} 3.5 \mathrm{I}$ & 3693.99 & $37^{87.5} 8$ & I 1095.08 \\
\hline 2 & 535.44 & 389.92 & 53 I. 65 & $\mathrm{I} 457.0 \mathrm{I}$ \\
\hline 3 & - & $9^{16.0 I}$ & 642.62 & I 558.63 \\
\hline \multirow[t]{2}{*}{4} & 一 & 一 & I $120.7^{\circ}$ & $1120.7^{\circ}$ \\
\hline & & & & 15231.42 \\
\hline
\end{tabular}


Provisions/Iooo

\begin{tabular}{|c|c|c|c|c|}
\hline Gen. & I & 2 & 3 & Total \\
\hline I & 6347.98 & 6098.18 & $5^{864} \cdot 4^{8}$ & I 8310.64 \\
\hline 2 & $845 \cdot 4.5$ & 814.16 & 779.I 7 & 2438.78 \\
\hline 3 & $\cdots$ & I $2 \mathrm{I} 9.4^{\circ}$ & 1179.37 & 2398.77 \\
\hline \multirow[t]{2}{*}{4} & $-\cdots-$ & - & 1954.16 & I954. I 6 \\
\hline & & & & $25 \mathrm{IO} 2.35$ \\
\hline
\end{tabular}

Difference between provisions and claims -.....-. -9870.93.

We determined that $70 \%$ of this value should be distributed amongst policy-holders.

$$
M=0.7 \times 9870.93=6909.66
$$

Participation values attributed to the various generations. $(I=\mathrm{I}, 2,3,4)$

\begin{tabular}{ccccc}
\hline $\mathrm{I}$ & $\mathrm{T}$ & 2 & 3 & 4 \\
\hline $\mathrm{F}(\mathrm{I})$ & 5040.18 & 671.29 & 660.28 & 537.9 \\
\hline
\end{tabular}

It would be interesting to compare the global practical result 9870.93 with the corresponding theoretical value obtained from the difference between the provisions and the credibility premiums, defined in 3.3 which is 9648.83 .

\section{Individual, Distribution}

In the following table we indicate the various generations of contracts, the values of the distributed surplus per policy and the corresponding number of policies that received them.

\begin{tabular}{|c|c|c|c|c|c|c|c|c|c|}
\hline Generation & Frequency & 5 & 25 & 37 & 108 & 217 & 437 & 620 & $5^{I}$ \\
\hline I & Surplus & 241.9 & $73 \mathbf{I} \cdot 5$ & I 527.4 & 2209.3 & 29 I I. I & 3644.8 & 4296.1 & o \\
\hline \multirow{2}{*}{2} & Frequency & 4 & 5 & 22 & 30 & 48 & 87 & - & 4 \\
\hline & Surplus & 613.9 & I 397.3 & $2 \mathrm{I} 40.2$ & 2882.3 & 3622.9 & 4186.7 & - & o \\
\hline \multirow{2}{*}{3} & Frequency & I & 7 & 36 & I 16 & I33 & - & 一 & 7 \\
\hline & Surplus & I 17.9 & 786.5 & I 516.5 & 2236.5 & 2650.7 & - & - & o \\
\hline \multirow{2}{*}{4} & Frequency & 4 & IOI & 389 & $\ldots$ & - & - & - & 6 \\
\hline & Surplus & 213.8 & $879 \cdot 4$ & I 175.8 & - & - & - & - & o \\
\hline
\end{tabular}


Analysing this table we can see on one hand the reduced number of policies causing losses (68 in 2500), thus not receiving any distributed surplus, and on the other, the high number of policies that received the mean annual value of about Iooo escudos.

If we notice that the value of $\pi$ for the portfolio was I 438.2 and that $70 \%$ of $\pi$ is I006.7, we can say that a great number of policies received the value corresponding to the excess premium paid out.

We can further conclude from this table the fact that a great number of policies with a mean annual participation lower than $0.7 \times \pi$ which under a classical premium scheme would have shown substantial losses.

6. We can finally conclude that a system of provisions of the type that was described lead to more satisfactory results both for the insurance company and for the policy-holder, doing away with the establishing of premiums "a priori" with which we can not agree, especially for risks whose parameters are practically unknown. 